\title{
Gender differences in pharmacokinetics, mass balance and phototoxicity of single-dose intravenous hemoporfin in healthy volunteers
}

\author{
Junyu $\mathrm{Xu}^{1}$, Xia Zhao ${ }^{1}$, Yan $\mathrm{Wu}^{2}$, Ping Tu${ }^{2}$, Peihong Sun ${ }^{1}$, Ying Zhou ${ }^{1}$, Xiaoyan Chen $^{3}$, Yifan Zhang ${ }^{3}$, \\ Jining $\mathrm{Tao}^{4}$, Yimin $\mathrm{Cui}^{1}$
}

${ }^{I}$ Department of Pharmacy, Peking University First Hospital, China, ${ }^{2}$ Department of Dermatology and Venereology, Peking University First Hospital, China, ${ }^{3}$ Shanghai Institute of Materia Medica, Chinese Academy of Sciences, China, ${ }^{4}$ Shanghai Fudan-Zhangjiang Bio-Pharmaceutical Co, Ltd, China

Background: Hemoporfin injection is for the treatment of port wine stain (PWS). It is a new porphyrin-related photosensitizer, which can be activated by light. The pharmacokinetics studies had been completed in our lab. Gender differences were observed in the pharmacokinetic parameters, and the fraction excreted unchanged in urine was less than $0.2 \%$. In order to investigate gender differences in larger sample sizes and the mass balance in healthy subjects with a single dose of intravenous hemoporfin, we conducted this study. The duration of phototoxicity was also observed after administration.

Methods: 16 healthy subjects were enrolled, male and female. They were randomly divided into two groups and received hemoporfin $5 \mathrm{mg} / \mathrm{kg}$. Fecal samples were collected only in the second group. The concentrations of hemoporfin (MHD) and its major metabolites, haematoporphyrin (HP) in plasma, urine and fecal were determined by LC-MS/MS. The pharmacokinetic parameters were calculated by WinNonlin. The skin reactions were observed by irradiating the selected part of the skin with a solar simulator, a KTP532 laser, an outdoor sunlight (only the second group of subjects receiving daylight) before administration and at the planned time points post-dosing.

Results: The main pharmacokinetic parameters of hemoporfin and haematoporphyrin were as table below. The half-life, the mean maximal concentration and the mean area under the concentration-time curve values showed no significant differences $(\mathrm{P}>0.05)$ in male and female subjects. 96-hour cumulative urinary excretion rate of unchanged drug accounted for only $0.132 \%(\mathrm{n}=15)$. Concentration of $\mathrm{HP}$ in urine was less than $10 \%$, without a quantitative analysis. 96hour cumulative fecal excretions rate of MHD and HP were 45.3\% and 1.05\% ( $\mathrm{n}=8)$. All subjects had no significant skin reaction at the site of irradiation by the solar simulator, the KTP532 laser and the outdoor sunlight.

Conclusions: No significant differences were observed in pharmacokinetics between male and female subjects after single intravenous hemoporfin. Parent drug and its major metabolites were excreted mainly in the faeces. After administration 52 hours, three kinds of light source do not cause phototoxicity. 